\title{
El Individuo en el contexto organizacional y su relación con las tecnologías de la información y comunicación
}

The individual inside the organizational context and its relation to information and communication technologies

\section{Luciane de Fátima Beckman Cavalcante (1) Marta Lígia Pomim Valentim (2)}

(1) UNESP - Universidad Estadual Paulista Júlio de Mesquita Filho, Facultad de Filosofía y Ciencias - Campus de Marília, Avenida da Saudade, 1183 B - Mirante, Marília SP Brasil, lucifbc@gmail.com,

(2) Campus Universitário - Cidade Universitária - Marilia, SP - Brasil, Caixa Posta 181, valentim@marilia.unesp.br

\begin{abstract}
Resumen
Este artículo presenta y discute los conceptos encontrados en la literatura sobre el individuo en el contexto de las organizaciones empresariales, aborda también la relación de los individuos con las tecnologías de información y de comunicación en ese tipo de ambiente. Presenta el análisis de los datos a partir de la aplicación de un cuestionario a los empleados de una empresa privada, en la que se analizaron, en escala de la importancia, los siguientes elementos: tecnología, información y personas.
\end{abstract}

Palabras-Clave: Organizaciones. Comportamiento Informacional. Tecnologías de la Información y Comunicación.

\section{Introducción}

Los individuos, directa o indirectamente, se relacionan o pertenecen a algún tipo de organización. Desde su nacimiento, el individuo es aprendido por distintos tipos de organización (familia, escuela, empresa, ejército, asociación de amigos etc.) y por las normas instituidas por la propia sociedad, debiendo, por sus actos y su trabajo, encontrar un lugar en que los otros lo reconozcan, como argumenta Enríquez (1966, p. 65).

Se sabe que las organizaciones están compuestas por infraestructura, tecnologías y personas. Sin embargo, entre estos tres elementos, el presente artículo considera las personas el elemento principal, teniendo en cuenta que es directa o indirectamente por ellas, las personas, que ocurre el desarrollo de todos los procesos organizacionales y el funcionamiento adecuado de las tecnologías adoptadas y o implantadas por la organización.

En ese contexto, cabe recordar que las tecnologías de información y comunicación (TICs) son relevantes, toda vez que el uso de

\begin{abstract}
This article presents and discusses the concepts found in the literature about the individual in the context of business organizations. It also addresses the relations between individuals and information and communication technologies within this environment. It presents the data analysis from the application of a questionnaire among the employees of a private company, in which the following elements were analyzed on a scale of importance: technology, information and people.
\end{abstract}

Keywords: Organizations. Information behavior. Information and communication technologies.

dichas tecnologías según Medeiros y Sauvé (2003, p.6) "[...] permite una infinidad de opciones de aplicaciones que pueden ayudar desde la automatización de tareas hasta la toma de decisiones gerenciales". Sin embargo, la tecnología no se hace sola, tampoco se desarrolla sola. Necesariamente necesita directa o indirectamente de la acción humana para su desarrollo, aplicación y uso. Se entiende que de nada vale implantar las TICs en el contexto organizacional, si el factor humano base y elemento estimulador de procesos y flujos organizacionales no aprovecha de la mejor forma este recurso.

\section{El individuo en las organizaciones}

El individuo no es ajeno al ambiente que lo rodea, tampoco al modo en que la organización y la cultura interfieren en su comportamiento y en la manera en que este individuo interpreta esa realidad.

Los individuos que pertenecen a una organización están sujetos a la cultura organizacional existente, la cual es la base que mueve las acciones de los individuos, por medio de valores, creencias, mitos, historias etc., los cuales son 
compartidos por los miembros de la organización, construyendo así el ambiente organizacional.

De acuerdo con Palazolli $(2000$, p. 1) la relación de influencia del comportamiento organizacional y de información de ese ambiente, ocurre entre individuo y organización, por lo tanto hay una relación de cambio.

[...] el trabajador crea vínculos afectivos positivos con el trabajo (satisfacción y compromiso) y desarrolla concomitantemente, afectos positivos hacia la organización (compromiso afectivo), notable cuando el empleado percibe que la organización se preocupa por su bienestar (percepción de suporte), retribuye sus contribuciones (percepción de reciprocidad) y, además consigue evaluar sus pérdidas caso haya ruptura de las relaciones de trabajo (comprometimiento calculado).

Al abordar el ideal de las organizaciones contemporáneas Freitas $(2005$, p. 109) argumenta que:

La imagen que ella transmite es de grandeza, omnipotencia, consenso, perfección, lugar de realización de deseos y de las expectativas de sus miembros y del público externo. Esa imagen será vivida, aúnque parcialmente, por los miembros como una creencia y una ilusión que apaciguan y acunan el deseo de sentirse seguro, protegido, amado y prestigiado.

De ese modo, muchas veces el individuo se ue a esa imagen como un medio de motivación para la realización de sus actividades. En lo que dice respecto a las personas, Davis y Newstron (1992, p. 10-11) exponen que existen cuatro conceptos básicos, los cuales caracterizan la naturaleza humana:

Diferencias Individuales: Las personas tienen mucho en común, pero cada persona en el mundo es también individualmente distinta.

La persona como un todo: Los diferentes rasgos humanos pueden ser estudiados separadamente, pero en un análisis final, todos se constituyen parte de un sistema que construye el todo de la persona.

El comportamiento motivado: La Psicología enseña que el comportamiento normal tiene ciertas causas. Ellas pueden estar relacionadas con las necesidades de la persona y o las consecuencias que resultan de sus acciones. La motivación es esencial al funcionamiento organizacional. No importa cuanta tecnología y equipo una organización tenga, pues ellas no pueden ser colocadas en uso a menos que sean liberadas y guiadas por personas motivadas a eso.

Valor de la persona (dignidad humana): Este concepto es de naturaleza distinta de los otros tres porque es más una filosofía ética de que una conclusión científica. Él sostiene que las personas deben ser tratadas de forma diferente de los otros factores de producción porque ellas pertenecen a al orden superior del universo. El concepto de dig- nidad humana rechaza la vieja idea de usar los empleados como instrumentos económicos.

De ese modo, en la visión de esos autores, el ambiente de trabajo de la organización está sujeto a todos los elementos que rodean a sus actores organizacionales, demostrando lo cuan importante y necesario que el ser humano es, y proporciona una comprensión profundizada sobre los aspectos que los abarcan, propiciando una mejor gestión no solo de los procesos organizacionales, sino y principalmente de los individuos que actúan en la organización.

Capra (1982, p. 40) defiende que "[...] sistema es un todo integrado cuyas propiedades no pueden ser reducidas a las de sus partes". Se percibe que como sistemas sociales, las organizaciones también engloban internamente otros sistemas que interfieren en los demás y viceversa, por cuanto están interligados, e individuos y organización deben trillar sobre una relación de reciprocidad.

\section{Comportamiento de Información en Ambientes Empresariales}

Las organizaciones están entramadas por intensos flujos de informaciones, según Moresi (2000, p.14), "[...] la importancia de la información para las organizaciones es universalmente aceptada, constituyendo [...] por lo menos uno de los recursos cuya gestión y aprovechamiento están directamente relacionados con el éxito deseado".

En empresas industriales, como es el caso de la empresa participante de la presente investigación, la información aunque no incorporada directamente al producto, puede ser aun entendida como insumo para el proceso productivo, una vez que es un elemento de conexión y coordinación entre las diversas áreas necesarias para la producción.

De ese modo, la información directa o indirectamente pasa por todos los procesos organizacionales, lo que denota relevancia de comprenderse como el comportamiento en relación a la información puede influir en el contexto organizacional.

En ese sentido, el comportamiento de información se relaciona con todo comportamiento que un individuo expresa en relación a la información. Davenport y Prusak (1998, p. 110) argumentan que el comportamiento de información está presente en todas las actitudes en relación a la información, su búsqueda, recuperación, filtración, uso y diseminación. 
Wilson (2000, p. 49) define comportamiento de información como [...]

la totalidad del comportamiento humano en relación a las fuentes y canales de información, incluyendo la búsqueda de información activa y pasiva, además del uso de la información.

Woida y Valentim (2008, p. 95-96) argumentan que el comportamiento de información está relacionado con la cultura de información existente en la organización:

[...] el comportamiento de información es resultado de la incorporación y de la práctica de elementos de la cultura de información existentes en la organización que valora la información, así como propicia que ese valor sea transformado en acciones de búsqueda, captura, adquisición, compartimiento y uso para la toma de decisiones.

Por la afirmación antedicha, se comprende que el comportamiento de información es algo intrínseco a cada organización. Sin embargo, a veces los gestores no prestan atención adecuada a esta cuestión, toda vez que la visión de la información como factor estratégico al desarrollo organizacional no siempre es vista de esa manera $y$, muchas veces, es incluso negada.

Mejorar el comportamiento de los individuos con relación a la información trae beneficios concretos, permitiendo eliminar barreras organizacionales para el cambio de informaciones y conocimiento útiles para los procesos de negocio y evitar ineficiencias causadas tanto por la sobrecarga cuanto por la dificultad de obtención de informaciones valiosas que permiten quedan restringidos a "feudos informacionales" (Beal, 2008, p. 45-46).

Se evidencia por la argumentación de Beal que una gestión con enfoque en el comportamiento de información de los individuos propicia beneficios que ayudan tanto a lidiar con la sobrecarga de información procedente de los intensos flujos de informaciones que rodean a la organización, cuanto a limitar comportamientos de posesión de la información en la cual la información es vista como poder por los individuos, hecho este que los llevan a no compartir y/o diseminar la información a los demás miembros de la organización.

\section{Tecnologías de Información y Comunicación}

Las sociedades han pasado por tres "períodos" evolutivos, a saber: la Revolución Agrícola, la Revolución Industrial y la Revolución Tecnológica. La cantidad de información resaltada de las revoluciones, hizo el que fuese inevitable el desarrollo de las TICs.
De ese modo, antes de los avances tecnológicos se produce la necesidad de adecuación de las organizaciones a las tecnologías en un escenario, cada vez más competitivo como abordan Fadel y Moraes (2005, p. 37).

Otra cuestión relevante a ser abordada cuando se trata de las TICs se refiere al hecho de que la tecnología no se hace sola, sino que depende, en la mayoría de las veces, del agente humano para su real desarrollo. Por tal hecho, es necesario un comportamiento favorable a su uso efectivo, pues según McGee y Prusak (1995, p. 4), "[...] no es la tecnología, sino su uso lo que crea valor adicional". Resende y Abreu (2003, p. 77) argumentan que todos "Ios componentes de la TI, interactúan y necesitan del componente fundamental que es el recurso humano, peopleware o humanware". Por lo tanto, se entiende que es por la interacción armónica del factor humano, junto a la información y las tecnologías que la organización puede conseguir administrar sus procesos y desarrollarse con éxito.

Molina y Valentim (2008) argumentan que "[...] con el desarrollo de las TICs, la información y el conocimiento pueden ser obtenidos por intermedio de diversas fuentes, almacenados en diferentes medios electrónicos y digitales". Por lo tanto, analizar esa relación del comportamiento de información junto a las TICs es de extrema importancia para las organizaciones que pretendan seguir competitivas en el escenario globalizado.

Chiavenato (2003, p. 576) argumenta que la tecnología de la información "[...] invade la vida de las organizaciones $y$ de las personas provocando profundas transformaciones". Las transformaciones sufridas con la adopción de las TICs ocurren a nivel general en la organización, sea afectando su estructura, sea afectando los individuos, visto que éstos pasan por todo un proceso de adaptación a la tecnología. De ese modo, adoptar cualquier tecnología en una determinada organización, implica la capacitación de los sujetos en la utilización de dicha tecnología.

Se evidencia que las TICs son herramientas importantes para que los gestores lidien con los intensos flujos de información en los cuales la organización está envuelta, visto que tales tecnologías pueden contribuir a dinamizar de la información en ese contexto.

Se percibe que la evolución tecnológica en sí misma es fruto de un proceso cultural. Con el paso de los años, las tecnologías fueron evolucionando cada vez más, implicando la 
adecuación de las personas en el de las tecnologías, una vez que éstas, directa o indirectamente están presentes en la cotidiana de los individuos.

Existe una variedad de tecnologías que pueden ayudar en el procesamiento y gerencia de las informaciones en el ámbito organizacional, así como en procesos desarrollados en ese contexto.

En el contexto organizacional, en lo que se refiere al comportamiento de información, las tecnologías de la información y comunicación son importantes para el individuo, ya que amplían las posibilidades de fuentes de información, bien y propician que la información sea compartida y diseminada de forma más rápida y ágil.

Sin embargo, la funcionalidad de las tecnologías está relacionada con el modo en que los individuos se adaptan a ellas, y las utilizan en la búsqueda, acceso y compartir de información que propicien la toma de decisiones más asertivas.

\subsection{Interacción entre Individuos y las TICs}

Como hemos visto la evolución tecnológica es un proceso cultural y natural del desarrollo de las sociedades. Sin embargo, se resalta que algunos casos es un proceso que implica en mudanzas y adaptaciones y, en muchos casos, no ocurre naturalmente. De ese, modo, se argumenta que sin determinada competencia en la utilización de las TICs, su efectividad puede verse perjudicada.

En las organizaciones empresariales, el advenimiento de las TICs, en la mayoría de las veces, es visto con temor por los individuos, hecho que puede ser generado por diversos factores tales como una cultura organizacional negativa en relación a las TICs. "EI impacto de la $\mathrm{TI}$ sobre el trabajo dependerá de la interacción entre los individuos y la tecnología" (Tavares, Costa, 2008, p. 12). En ese sentido, es posible percibirse lo cuan importante es el factor humano a la efectividad de las TICs.

La tecnología de la información tendrá utilidad real a partir del momento en que sea infundido en los individuos que dicha tecnología contribuye y ofrece posibilidades que permiten el acceso, la búsqueda y el compartimiento de informaciones en determinados contextos para determinados fines.

En el contexto organizacional, la cultura organizacional puede ser un elemento influya en el individuo, en relación al comportamiento de información dirigido al uso de las tecnologías de la información y la comunicación en el entorno de la organización. Así, la cultura organizacional es un factor extremamente importante, por ser una influencia clave en comportamientos y actitudes, puede condicionar excesivamente la relación del sujeto organizacional con las TICs en ese contexto.

Fialho et al. (2006, p. 61) argumentan que el “[...] énfasis exagerado en la tecnología de la información puede llevar a la negligencia de otras fuentes de información y las empresas raramente identifican que el comportamiento y la cultura deben cambiar para que la tecnología sea exitosa" (Fialho et al., 2006, p. 62).

Sin embargo, trabajar una cultura que propicie comportamientos positivos en relación al uso de las TICs es una tarea delicada, visto que implica en proceso de mudanza y reestructuración de la cultura organizacional, lo que lleva tiempo y, principalmente, exige la participación de todos los trabajadores de la empresa. Obviamente actualmente las TICs se hacen presentes en la realidad organizacional, una vez que se convierten en parte del proceso evolutivo de cada organización.

Las TICs son, por lo tanto, un factor importante del contexto organizacional, visto que dinamizan la información en tal contexto. Ellas ayudan a establecer un eslabón entre la información y las personas, tanto en relación al acceso cuanto en relación al uso de la información en el contexto organizacional.

Sin embargo, la efectividad de las TICs en las organizaciones depende de un elemento preponderante, al cual muchas veces no se le da la debida atención, que es el ser humano. Todos los procesos y tareas organizacionales pasan directa o indirectamente por la acción humana, sufren interferencia de la acción humana y con las TICs este hecho no es diferente. Es decir, se debe enfocar la información y las personas en el contexto organizacional antes de mejorar determinados sistemas basados en tecnología.

\section{Procedimientos metodológicos y resultados}

Se buscó evaluar la importancia que la empresa investigada otorgaba a los siguientes elementos: tecnología, información y personas. Para ello, se aplicó un cuestionario a 20 empleados de una empresa privada de fabricación de celulosas, localizada en el interior del Estado de São Paulo, Brasil. De ese modo, los 20 participantes tenían que indicar, a partir 
de sus percepciones, la importancia relativa de la tecnología, la infomación o las personas usando la escala 1,2 y 3 , siendo (1) más importante y (3) menos importante.

\subsection{Resultados}

Se verificó que del total de 20 sujetos participantes en la investigación, el $80 \%$ indicó que la empresa considera las personas más importantes y el $63 \%$ indicó que la tecnología es menos importante, como es posible observar en la Figura 1 y Figura 2:

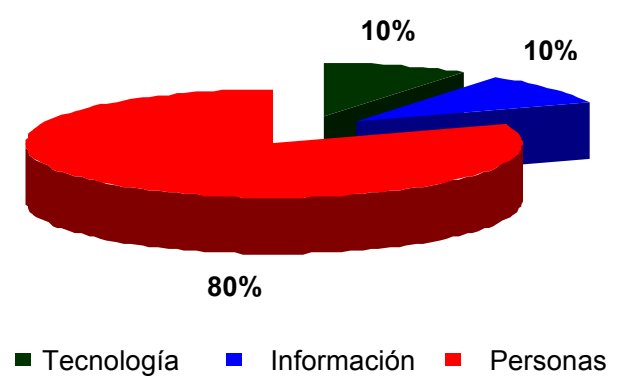

Figura 1. Más importante.

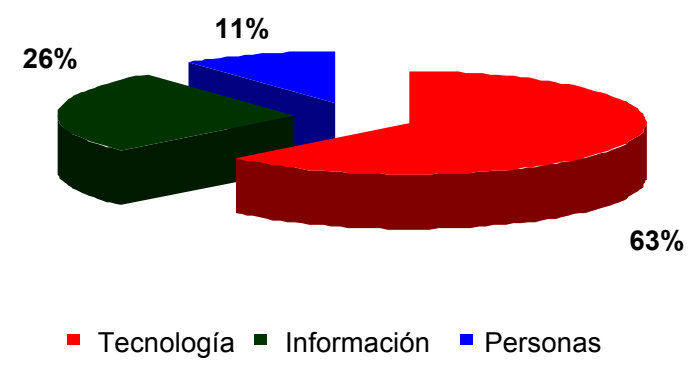

Figura 2. Menos Importante.

Por medio de la Figura 1 se observa que entre tecnología, información y personas, aproximadamente el $80 \%$ de las respuestas indicaron que las personas son más importantes. Este es un dato muy significativo, pues denota que las personas sienten que son parte da empresa y saben que son reconocidas por ella.

Bajo el punto de vista del comportamiento de información este dato es extremamente relevante, pues la valoración de la persona es un paso importante para motivarla, toda vez que desarrolla la voluntad de colaborar con posibles cambios y adecuaciones, así como propicia que se vuelvan más abiertas para expresar las necesidades de información y compartir información.
Se infiere a partir de lo demostrado en la Figura 2 , que entre tecnología, información y personas la menos importante, según aproximadamente el $63 \%$ de los encuestados, es la tecnología, pese a que actúan como herramientas importantes para el desarrollo de actividades y tareas en el contexto organizacional.

Este es un dato extremamente significativo, porque demuestra que aun con los recursos tecnológicos, lo que todavía prevalece en la empresa investigada son las personas, y las personas tienen consciencia de eso, comprobando el argumento encontrado en la literatura de que el impacto de la $\mathrm{TI}$ en una organización depende de la interacción entre los individuos y la tecnología.

En lo que se refiere al comportamiento de información, se evidencia que, en detrimento de la tecnología, quien hace y maneja la búsqueda, el acceso, el compartir y el uso de la información son las personas; o sea, pese a la importancia de las tecnologías, éstas son consideradas una herramienta que ayuda en el desempeño de los procesos organizacionales.

Se concluye, a partir de los datos colectados y analizados, que la cultura organizacional de la empresa investigada es una cultura que valora el individuo como colaborador del desarrollo organizacional, y aunque la información se considera un elemento importante para los procesos organizacionales, la atención de la empresa se vuelve primeramente para sus empleados, siendo estos considerados los más importantes.

\section{Conclusión}

En suma, las personas son vistas como elemento principal de la organización, toda vez que es por ellos que se realizan todos los procesos organizacionales, así como la información. Son las personas las que hacen que las tecnologías de la información y la comunicación tengan real efectividad en el contexto organizacional.

En ese sentido, se destaca que el uso eficaz de las TICs ayuda al comportamiento de información de los individuos. Sin embargo, solamente traerá resultados concretos si la cultura de información propicia la diseminación de valores y creencias acerca de la importancia de las tecnologías, que, una vez compartidas entre los actores organizacionales, desarrollen un comportamiento positivo en relación a ellas.

Por lo expuesto, siendo la información un factor que sobrepasa todo el contexto organizacional, asi como todas las actividades desarrolladas en 
este contexto, la clave para su gerencia está íntimamente conectada con comprender y administrar el comportamiento de información de los individuos que actúan en la organización, toda vez que, como argumentan Davenport y Prusak (1998), la información y el conocimiento son creaciones esencialmente humanas. De ese modo, no es posible administrarlos sin considerar el papel fundamental desempeñado por las personas en ese universo.

\section{Referencias}

Beal, A. (2004). Gestão estratégica da informação: como transformar a informação e a tecnologia da informação em fatores de crescimento e de alto desempenho nas organizações. São Paulo: Atlas, 2004.

Chiavenato, I. (2003). Introdução à teoria geral da Administração: uma visão abrangente da moderna administração das organizações. Rio de Janeiro: Elsevier, 2003.

Capra, F. (1982). O ponto de mutação: a ciência, a sociedade e a cultura emergente. São Paulo: Cultrix, 1982. 447 p.

Choo, C. W. (2006). A empresa do conhecimento: como as organizações usam a informação para criar significado, construir conhecimento e tomar decisões. São Paulo: Senac, 2006.

Davenport, T. H.; Prusak, L. (1998). Ecologia da informação: por que só a tecnologia não basta para o sucesso na era da informação. São Paulo: Futura, 1998.

Davis, K.; Newstrom, J. W. (1992). Comportamento humano no trabalho. São Paulo: Pioneira, 1992.

Enriquez, E. (1966). Imaginário social, recalcamento e repressão nas organizações locais. // Colóquio Organizations da ARIP, jun. 1966.
Fadel, B.; Moraes, C. R. B de (2005). As ondas de inovação tecnológica. // FACEF Pesquisa, Franca. 8:1 (2005) 3440.

Fialho, J. F.; Andrade, M. E. A. (2007). Comportamento informacional de crianças e adolescentes: uma revisão da literatura estrangeira. // Ciência da Informação, Brasília. 36:1 (jan./abr. 2007) 20-34.

Fialho et al.(2006). Gestão do Conhecimento e aprendizagem: as estratégias competitivas da sociedade pósindustrial. Florianópolis. Visual Books 2006.

Freitas, M. E. (2005). Cultura organizacional: identidade, sedução e carisma?. Rio de Janeiro: Editora FGV, 2005.

Medeiros, E. M. S. De; Suavé, J. P. (2003). Avaliação do impacto de tecnologias da informação emergentes nas empresas. Rio de Janeiro: Qualiymark, 2003.

Mcgee, J. V.; Prusak, L. (1995) Gerenciamento estratégico da informação. Rio de Janeiro: Campus, 1995.

Moresi, E. A. D. (2000). Delineando o valor do sistema de informação de uma organização. // Ciência da informação. Brasília. 29:1 (jan./abr. 2000).

Palazolli, F. (2000). Percepção de justiça nas organizações como antecedentes dos comportamentos de cidadania organizacional. // Caderno de Pesquisas em Administração. São Paulo. 7:3 (julho/setembro, 2000).

Tavares, E.; Costa, I. de S. A. (2008). O papel dos valores individuais na interação entre indivíduos e tecnologia de informação. // Revista Portuguesa e Brasileira de Gestão. (2008).

Valentim, M. L. P.; Gelinski, J. V. (2006). Gestão do conhecimento corporativo. // Valentim, M. L. P. (Org.). Informação, conhecimento e inteligência organizacional. Marília: FUNDEPE Editora, 2006. 115-131.

Walton, R. E. (1994). Tecnologia de informação: o uso de tecnologia de informação pelas organizações que obtém vantagem competitiva. São Paulo: Atlas, 1994

Recibido: 16-04-2010. Aceptado: 12-07-2010. 